\title{
Review
}

\section{Evidence Map of Cupping Therapy}

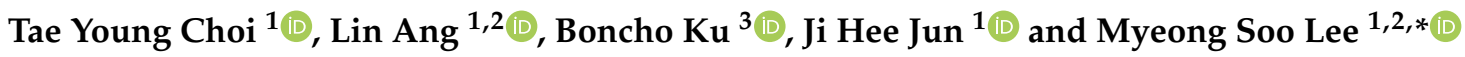 \\ 1 Clinical Research Division, Korea Institute of Oriental Medicine, Daejeon 34054, Korea; \\ superoung@kiom.re.kr (T.Y.C.); anglin2808@kiom.re.kr (L.A.); zhixi04@kiom.re.kr (J.H.J.) \\ 2 Korean Convergence Medicine, University of Science and Technology, 217, Gajeong-ro, Yuseong-gu, \\ Daejeon 34113, Korea \\ 3 Clinical Research Coordinating Team, Korea Institute of Oriental Medicine, Daejeon 34054, Korea; \\ secondmoon@kiom.re.kr \\ * Correspondence: drmslee@gmail.com or mslee@kiom.re.kr; Tel.: +82-42-868-9266; Fax: +82-42-863-9299
}

\section{check for}

updates

Citation: Choi, T.Y.; Ang, L.; Ku, B.; Jun, J.H.; Lee, M.S. Evidence Map of Cupping Therapy. J. Clin. Med. 2021, 10, 1750. https://doi.org/10.3390/ jcm10081750

Academic Editor: Younbyoung Chae

Received: 13 March 2021

Accepted: 15 April 2021

Published: 17 April 2021

Publisher's Note: MDPI stays neutral with regard to jurisdictional claims in published maps and institutional affiliations.

Copyright: (c) 2021 by the authors. Licensee MDPI, Basel, Switzerland. This article is an open access article distributed under the terms and conditions of the Creative Commons Attribution (CC BY) license (https:// creativecommons.org/licenses/by/ $4.0 /)$.

\begin{abstract}
This study aimed to describe and assess the current evidence in systematic reviews on cupping therapy for various conditions. We searched PubMed, EMBASE, Cochrane Database of Systematic Reviews, China National Knowledge Infrastructure, and six Korean databases for systematic reviews of trials on cupping treatments for any condition published prior to March 2021. We used a bubble plot to graphically display the clinical topics, the number of articles, the number of participants in the total population, confidence, and effectiveness. Thirteen systematic reviews that met the inclusion criteria were included in the evidence map, and 16 bubbles were created. The findings from six reviews showed potential benefits of cupping for conditions such as low back pain, ankylosing spondylitis, knee osteoarthritis, neck pain, herpes zoster, migraine, plaque psoriasis, and chronic urticaria. Cupping has been applied in a variety of clinical areas, and systematic reviews in a few of these areas have demonstrated statistically significant benefits. The evidence map provides a visual overview of cupping research volume and findings. Evidence mapping can facilitate the transfer of knowledge from researchers to policymakers and promote research on musculoskeletal pain (such as low back pain, neck pain, and knee osteoarthritis) and skin disease (plaque psoriasis).
\end{abstract}

Keywords: evidence map; cupping therapy; systematic review; evidence synthesis

\section{Introduction}

Cupping has been used with traditional and alternative medicine to treat a variety of conditions in China, Korea, and East Asia. In Taiwan, $12.8 \%$ of participants in a previous study reported undergoing cupping therapies in the past year [1]. Cupping has also recently gained popularity in Western countries such as Europe, the United States, and Arabia [2]. Although it is a timeworn technique, it is still being used in the treatment of various medical conditions [3], especially those involving pain, such as low back pain [4], neck pain [5], and knee osteoarthritis [6].

Cupping, whether it is performed dry or wet, is a technique in which cups made of plastic, bamboo, or glass cups are used in suctioning the skin over acupuncture points, painful areas, or reflex zones [7]. For dry cupping, the cup creates a mild vacuum on the skin to aggravate the subcutaneous tissues without blood being drawn. For wet cupping, the cup suctions the lacerated skin to draw blood from the dermal microcirculation. Cupping has also been used to improve subcutaneous blood flow to skin and muscles and to stimulate the autonomic nervous system [7,8]. Besides, cupping is also used for draining excess fluids and toxins, loosening adhesions, and lifting connective tissues [8]. Correspondingly, in the theory of traditional medicine, cupping promotes the circulation of Qi and blood of the treatment area to alleviate pain and tension caused by stagnation, and expels the pathogenic factors, eventually leading to the restoration of physiological harmony and balance.

The usage of cupping therapies to improve health outcomes has been increasing continuously, resulting in cupping being the subject of investigation in many primary 
studies and systematic reviews (SRs). However, previous research has covered a variety of conditions, populations, and settings. Several SRs have investigated the therapeutic effect of cupping therapy for low back pain [9], neck pain [10], and hypertension [11]. An overview of SRs identified 8 reviews published in 2015 and concluded that cupping may aid in pain-related conditions, acne, and facial paralysis [12]. However, previous reviews are outdated and reported in insufficient detail pertaining to the included studies. Moreover, they did not use graphical representations, which facilitate the interpretation of results.

Evidence maps are new synthesis tools that involve searching systematically and characterizing existing research on a topic of interest for the identification of knowledge gaps and future research needs [13]. We conducted an evidence mapping analysis to determine the distribution of evidence available regarding the indication of cupping for various conditions as well as different forms of cupping therapy; this form of systematic literature synthesis visualized the volume and the topics of research.

This evidence map study aimed to present a visual overview of the distribution of evidence available on cupping therapy for various conditions, along with a concomitant narrative that will aid stakeholders in interpreting the state of the current evidence for informed policy and clinical decision making.

\section{Methods}

\subsection{Study Design}

There are no official standardized methods for evidence mapping [14]. The methods used in this study were based on those reported by Hempel et al., in the "Evidence Map of Acupuncture" [15].

\subsection{Electronic Searches and Search Strategy}

The following electronic databases were systematically searched for in literature published from their dates of inception to 16 March 2021: PubMed, EMBASE, the Cochrane Database of Systematic Reviews (CDSR), one Chinese database (the China National Knowledge Infrastructure (CNKI)), and six Korean medical databases (Research Information Service System (RISS), DBpia, Korea Med, Oriental Medicine Advanced Search Integrated System (OASIS), Korean Studies Information Services System (KISS), and Korean Medical Database (KM base)). In addition, the reference lists of the potentially eligible articles were searched manually to further identify additional eligible papers.

The search terms used were "systematic review" or "meta-analysis" and "cupping therapy", and the database-specific filters were used to search for SRs without language restrictions (Supplement 1).

\subsection{Inclusion Criteria}

\subsubsection{Design}

Only SRs focusing on cupping therapy, which compiled primary research studies of any clinical indications, were included. We defined SRs as self-identified review articles that included the phrase "systematic review" in the title or the main text and/or review articles that reported the sources systematically searched for in the identified studies. SRs are studies in which the findings of studies are systematically selected from the medical literature and are summarized for each type of intervention.

\subsubsection{Population}

Individuals with any conditions were included.

\subsubsection{Intervention}

SRs of the effectiveness of cupping for any clinical indication were eligible for inclusion. All trials were included regardless of the type of cupping and control intervention. Trials that used cupping as an adjunctive treatment were also included. 


\subsubsection{Outcomes}

SRs that report patient health outcomes were included and those of provider outcomes, study designs, or intervention features unrelated to patient health outcomes were excluded.

\subsubsection{Timing}

SRs that summarize the assessments of interventions of any duration with any followup period were included.

\subsubsection{Systematic Review Selection}

Formal SR articles with and without a meta-analysis related to any type of cupping therapy for any type of medical condition were eligible for inclusion. Articles that were not performed using systematic methods such as reviews, comments, and overviews, were excluded. Two reviewers (TYC and JHJ) independently screened all the titles and abstracts and read the full text of articles to exclude irrelevant SRs. Disagreements were resolved through discussion and consensus, and an additional reviewer (MSL) was consulted. The originals and updates of SRs by the same author group were always available, but only the most recent version was considered. In the case of multiple publications, they were considered as one review, and data were extracted from all the available publications. If multiple reviews of similar clinical topics were identified, the most pertinent and bestperformed SR was used for the inclusion of evidence maps selected based on the results of the AMSTAR 2 assessment.

\subsection{Methodological Quality Assessment}

The Assessing the Methodological Quality of Systematic Reviews (AMSTAR)-2 tool was used to critically appraise the quality of reporting for each included SR. A validated 16-item instrument was also used for the appraisal of the SRs based on critical flow and bias using ratings of "yes", "partial yes", or "no" [16]. The overall confidence in the results of the SRs was then judged using the following four categories: "high" for none or one noncritical weakness, "moderate" for more than one noncritical weakness, "low" for one critical flaw with or without noncritical weaknesses, and "critically low" for more than one critical flaw with or without noncritical weaknesses.

\subsection{Data Extraction}

Information regarding the population, intervention, comparison and outcomes (PICO) process, the numbers of randomized controlled trials (RCTs) included in each SR, summary effect estimates for the main outcomes, overall risk of bias of the included RCTs, publication bias, and conclusions as quoted from the original article, were extracted. The information for each intervention was extracted separately if the SR was designed as an overview or an umbrella review. All articles were read by two independent reviewers (TYC and $\mathrm{JHJ})$, and the data were extracted from the articles according to the predefined criteria for the data extraction and methodological quality assessments. Disagreements were resolved by consensus, and when necessary, an additional reviewer (MSL) participated in the discussion.

\subsection{Evidence Map Presentation and Domains}

We used topics of investigation of the included SRs to categorize them. The results of evidence mapping were presented using characteristic tables of included SRs and a graphical display of bubble plots. Each bubble in the chart represents the included evidence for clinical topics as assessed by the SR investigating the effectiveness of cupping.

The outcomes, populations, or clinical indications were focused in the reviews. The SRs were grouped into clinical topics by one reviewer, and the grouping results were discussed and reviewed by the team. The decisions to not combine potentially related topic areas were due to the lack of overlapping between studies included in the reviews and differences in the reported outcomes or the review's conclusion. When SRs contained 
various diseases, each disease was analyzed separately and used multiple times in the bubble plot. The evidence map used R software (version 3.5.0; R Foundation for Statistical Computing, Vienna, Austria).

This chart displays the information in four dimensions:

(1) X-axis: effect estimate

The clinical effectiveness of cupping therapy was evaluated based on the effect estimates and the overall risk of bias reported in the selected SRs. Clinical effectiveness was classified as "effective" when the effect estimates were significantly positive with a low overall risk of bias, "potentially effective" when the effect estimates were significantly positive with a high overall risk of bias, or "unclear" when the effect estimates were negative or the overall risk of bias was unclear.

(2) Y-axis: number of articles

The literature size was defined as the number of RCTs in the selected SRs on the topic. Although reviews often differ in their inclusion criteria, all reviews are likely to include studies of well-established research design, such as an RCT, and provides a broad estimate of the research volume.

(3) Bubble size: number of participants in the total population

The size of each SR bubble was proportional to the number of participants in the total sample size for the effects of cupping.

(4) Color: strength of the findings

Confidence was determined based on the results of the AMSTAR 2 assessment and was classified into the following four categories: yellow circles correspond to "high confidence"; green circles correspond to "moderate confidence"; blue circles correspond to "low confidence", or red circles corresponded to "critically low confidence".

\subsection{Narrative Synthesis}

To provide more details from the included SRs, a concomitant narrative synthesis was expanded upon the visual evidence map. Such details included descriptions of the findings, the features of cupping therapy, and the types of diseases.

\section{Results}

\subsection{Description of Included SRs}

\subsubsection{Selection Diagram}

The electronic database search identified 107 potentially relevant studies. After duplicate publications were removed, the titles and abstracts of 85 papers were screened. The full texts of 37 articles were reviewed for eligibility and 23 SRs were included in the review. According to the results of the AMSTAR 2 assessment, only the best performed SRs were selected for further inclusion (Figure 1). Finally, 13 SRs [9-11,17-25] that met the inclusion criteria were included in the evidence map, and 16 bubbles were created. The studies selected were conducted in 3 countries: Brazil $(n=1)$, China $(n=9)$, and Korea $(n=3)$. The information of the excluded 10 SRs information is presented in Supplements 2 and 3.

\subsubsection{Included Diseases}

The effectiveness, literature size, and confidence level for cupping therapy in the included SRs were identified and evaluated. A few SRs covered several types of diseases, which were assessed separately in our evaluation. The medical conditions studied in the RCTs of the included SRs were back pain $(n=7)$, ankylosing spondylitis $(n=2)$, knee osteoarthritis $(n=3)$, neck pain $(n=4)$, cervical spondylosis $(n=1)$, lateral femoral cutaneous neuritis $(n=1)$, scapulohumeral periarthritis $(n=1)$, herpes zoster $(n=4)$, facial paralysis $(n=1)$, acne $(n=1)$, cervical spondylosis $(n=1)$, stroke rehabilitation $(n=1)$, hypertension $(n=2)$, migraine $(n=1)$, plaque psoriasis $(n=2)$, chronic urticarial $(n=1)$, and obesity $(n=1)$. 

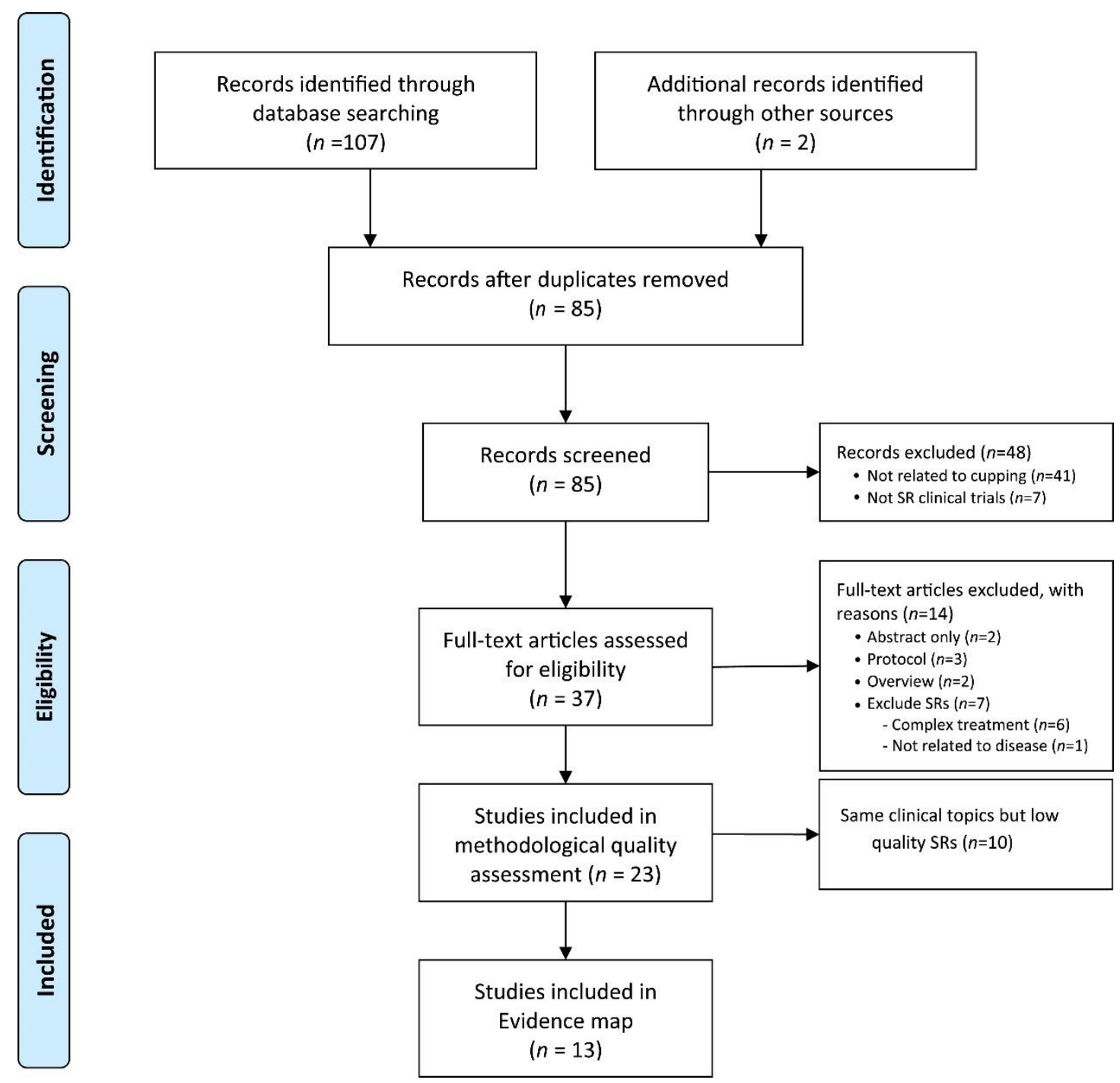

Figure 1. PRISMA diagram for the included studies. SR: systematic review.

\subsubsection{Intervention Components Described}

Given that there are many types of cupping therapies, accurate and detailed reporting about these interventions is necessary to understand which therapies are included and synthesized in the SRs. A description of the cupping style was included in all the SRs. Only two SRs focused on one type of cupping, which is wet cupping [11] and moving cupping [17], whereas the other SRs included all types of cupping. Cupping has been applied in a variety of clinical areas, and systematic reviews in a few of these areas have demonstrated statistically significant benefits (Table 1). 
Table 1. Summary of the included systematic reviews of cupping therapy.

\begin{tabular}{|c|c|c|c|c|c|c|c|c|}
\hline $\begin{array}{l}\text { First Author } \\
\text { (year) (Ref) } \\
\text { Country }\end{array}$ & $\begin{array}{l}\text { Condition } \\
\text { Search Date } \\
\text { No. of Primary Studies }\end{array}$ & $\begin{array}{l}\text { Cupping } \\
\text { Therapy }\end{array}$ & Comparator & Outcome & $\begin{array}{l}\text { Overall Risk } \\
\text { of Bias }\end{array}$ & $\begin{array}{l}\text { Effect Estimates for Main } \\
\text { Outcomes (Meta-Analysis) }\end{array}$ & $\begin{array}{l}\text { Conclusion } \\
\text { (Quoted from the } \\
\text { Original Paper) }\end{array}$ & $\begin{array}{l}\text { Overall } \\
\text { Confidence }\end{array}$ \\
\hline $\begin{array}{l}\text { Moura } \\
(2018)[9] \\
\text { Brazil }\end{array}$ & $\begin{array}{l}\text { Chronic back pain } \\
\text { May } 2018 \\
16 \text { RCTs }\end{array}$ & All types & $\begin{array}{l}\text {-Sham } \\
\text {-Waiting list } \\
\text {-WM } \\
\text {-None }\end{array}$ & Pain & High & $\begin{array}{l}\mathrm{MD}-1.59(-2.07,-1.10) \\
p=0.001\end{array}$ & $\begin{array}{l}\ldots \text { has shown } \\
\text { positive results ... }\end{array}$ & Effective \\
\hline $\begin{array}{l}\text { Kim } \\
(2018)[10] \\
\text { Korea }\end{array}$ & $\begin{array}{l}\text { Neck pain } \\
\text { Jan } 2018 \\
18 \text { RCTs }\end{array}$ & All types & $\begin{array}{l}\text {-Usual care } \\
\text {-AT } \\
\text {-Waiting list } \\
\text {-No treatment }\end{array}$ & $\begin{array}{l}\text { (1)Pain } \\
\text { (2)Function }\end{array}$ & High & $\begin{array}{l}\text { vs. no treatment } \\
\text { (1) MD }-2.42(-3.98,-0.86) \text {, } \\
p<0.00001 \\
(2) \mathrm{MD}-4.34(-6.77,-1.19) \text {, } \\
p=0.0005 \\
\text { vs. active control } \\
\text { (1) MD }-0.89(-1.42,-0.37) \text {, } \\
p=0.0009 \\
(2) \mathrm{MD}-4.36(-8.67,-0.04), \\
p=0.05\end{array}$ & $\begin{array}{l}\ldots \text { reduce neck } \\
\text { pain... }\end{array}$ & $\begin{array}{l}\text { Potentially } \\
\text { effective }\end{array}$ \\
\hline $\begin{array}{l}\text { Lu } \\
(2018)[11] \\
\text { China }\end{array}$ & $\begin{array}{l}\text { Hypertension } \\
\text { May } 2018 \\
7 \text { RCTs }\end{array}$ & Wet cupping & $\begin{array}{l}-\mathrm{WM} \\
-\mathrm{AT}\end{array}$ & $\begin{array}{l}\text { (1) SBP } \\
\text { (2) DBP } \\
\text { (3) } \\
\text { Antihypertensive } \\
\text { effect } \\
\text { (4) Effective rate }\end{array}$ & High & $\begin{array}{l}\text { (1) MD -2.24 }(-9.13,4.65) \\
p=0.52 \\
\text { (2) MD }-2.11(-8.85,4.64) \\
p=0.54 \\
\text { (3) RR } 1.09(0.99,1.20), p=0.07 \\
\text { (4) RR } 1.22(1.05,1.40), p=0.007\end{array}$ & $\begin{array}{l}\ldots \text { no firm } \\
\text { conclusions ... }\end{array}$ & Unclear \\
\hline $\begin{array}{l}\text { Ma } \\
(2018)[18] \\
\text { China }\end{array}$ & $\begin{array}{l}\text { Ankylosing spondylitis } \\
\text { Dec } 2017 \\
5 \text { RCTs }\end{array}$ & All types & $\begin{array}{l}\text {-Sham/placebo } \\
\text {-WM }\end{array}$ & $\begin{array}{l}\text { (1) BASFI } \\
\text { (2) BASDAI } \\
\text { (3) ESR }\end{array}$ & High & $\begin{array}{l}\text { (1) MD }-16.63(-17.75 \\
-15.51), p<0.00001 \\
(2) \mathrm{MD}-9.93(-10.34,-9.52) \\
p<0.00001 \\
(3) \mathrm{MD}-3.96(-4.69,-3.23) \\
p<0.00001\end{array}$ & $\begin{array}{l}\ldots \text { weak } \\
\text { evidence ... }\end{array}$ & $\begin{array}{l}\text { Potentially } \\
\text { effective }\end{array}$ \\
\hline $\begin{array}{l}\mathrm{Li} \\
(2017)[19] \\
\text { China }\end{array}$ & $\begin{array}{l}\text { Knee osteoarthritis } \\
\text { Jan } 2017 \\
7 \text { RCTs }\end{array}$ & All types & $\begin{array}{l}\text {-Sham/placebo } \\
\text {-WM }\end{array}$ & $\begin{array}{l}\text { WOMAC } \\
\text { (1) Pain } \\
\text { (2) Stiffness } \\
\text { (3) Physical } \\
\text { function }\end{array}$ & High & 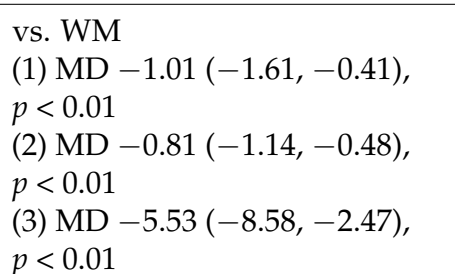 & $\begin{array}{l}\ldots \text { weak evidence } \\
\ldots \text { cupping } \\
\text { therapy ... }\end{array}$ & $\begin{array}{l}\text { Potentially } \\
\text { effective }\end{array}$ \\
\hline
\end{tabular}


Table 1. Cont.

\begin{tabular}{|c|c|c|c|c|c|c|c|c|}
\hline $\begin{array}{l}\text { First Author } \\
\text { (year) (Ref) } \\
\text { Country }\end{array}$ & $\begin{array}{l}\text { Condition } \\
\text { Search Date } \\
\text { No. of Primary Studies }\end{array}$ & $\begin{array}{l}\text { Cupping } \\
\text { Therapy }\end{array}$ & Comparator & Outcome & $\begin{array}{l}\text { Overall Risk } \\
\text { of Bias }\end{array}$ & $\begin{array}{l}\text { Effect Estimates for Main } \\
\text { Outcomes (Meta-Analysis) }\end{array}$ & $\begin{array}{l}\text { Conclusion } \\
\text { (Quoted from the } \\
\text { Original Paper) }\end{array}$ & $\begin{array}{l}\text { Overall } \\
\text { Confidence }\end{array}$ \\
\hline $\begin{array}{l}\text { Zhang } \\
(2017)[20] \\
\text { China }\end{array}$ & $\begin{array}{l}\text { Several conditions } \\
\text { Mar } 2017 \\
23 \text { RCT (Cervical } \\
\text { spondylosis, } 7 \text { RCTs; } \\
\text { lateral femoral cutaneous } \\
\text { neuritis, } 2 \text { RCTs; } \\
\text { scapulohumeral } \\
\text { periarthritis, } 2 \text { RCTs; } \\
\text { Others, } 12 \text { RCTs) }\end{array}$ & All types & $\mathrm{AT}$ & Effective rate & High & $\begin{array}{l}\text { Cervical spondylosis } \\
\text { RR } 1.13(1.01,1.26), p=0.04 \\
\text { Lateral femoral cutaneous } \\
\text { neuritis } \\
\text { RR } 1.10(1.00,1.22), p=0.71 \\
\text { Scapulohumeral periarthritis } \\
\text { RR } 1.31(1.15,1.51), p=0.84\end{array}$ & $\begin{array}{l}\text { Cupping ... safe } \\
\ldots \text { relieving pain. }\end{array}$ & Unclear \\
\hline $\begin{array}{l}\text { Cao }(2010)[21] \\
\text { China }\end{array}$ & $\begin{array}{l}\text { Herpes zoster } \\
\text { Feb } 2009 \\
8 \text { RCTs }\end{array}$ & Wet cupping & $\begin{array}{l}\text {-No treatment } \\
\text {-Placebo } \\
\text {-WM }\end{array}$ & Effective rate & High & $\begin{array}{l}\text { vs. WM } \\
\text { RR } 1.15(1.91,3.24), p=0.0 .005\end{array}$ & $\begin{array}{l}\ldots \text { appears to be } \\
\text { effective } \ldots\end{array}$ & $\begin{array}{l}\text { Potentially } \\
\text { effective }\end{array}$ \\
\hline $\begin{array}{l}\text { Cao }(2012)[22] \\
\text { China }\end{array}$ & $\begin{array}{l}\text { Several conditions } \\
\text { Dec } 2010 \\
135 \text { RCTs } \\
\text { Herpes zoster (15 RCTs) } \\
\text { Facial paralysis (15 RCTs) } \\
\text { Acne (6 RCTs) } \\
\text { Cervical spondylosis } \\
\text { (6 RCTs) } \\
\text { Other conditions } \\
\text { (93 RCTs) }\end{array}$ & All types & $\begin{array}{l}-\mathrm{WM} \\
-\mathrm{AT}\end{array}$ & Effective rate & High & $\begin{array}{l}\text { vs. WM } \\
\text { Herpes zoster } \\
\text { RR } 2.07(1.77,2.43), p<0.00001 \\
\text { Facial paralysis } \\
\text { RR } 1.49(1.35,1.65), p<0.00001 \\
\text { Acne } \\
\text { RR 2.14 }(1.40,2.65), p=0.0003 \\
\text { Cervical spondylosis } \\
\text { RR } 2.07(1.77,2.43), p<0.00001\end{array}$ & $\begin{array}{l}\text { No confirm } \\
\text { conclusion } \ldots\end{array}$ & Unclear \\
\hline $\begin{array}{l}\text { Lee }(2010 a)[23] \\
\text { Korea }\end{array}$ & $\begin{array}{l}\text { Stroke rehabilitation } \\
\text { Mar } 2010 \\
5 \text { studies } \\
\text { (3 RCTs, } 2 \text { UOS) }\end{array}$ & All types & $-\mathrm{AT}$ & $\begin{array}{l}\text { (1) Effective rate } \\
\text { (2) VAS }\end{array}$ & High & $\begin{array}{l}\text { (1) } p<0.05 \\
\text { (2) } p=0.004\end{array}$ & Insufficient ... & Unclear \\
\hline $\begin{array}{l}\text { Seo } \\
(2018)[24] \\
\text { Korea }\end{array}$ & $\begin{array}{l}\text { Migraine } \\
\text { Sep } 2016 \\
7 \text { RCTs }\end{array}$ & All types & $\begin{array}{l}-\mathrm{WM} \\
-\mathrm{AT}\end{array}$ & $\begin{array}{l}\text { (1) Effective rate } \\
\text { (2) VAS }\end{array}$ & High & $\begin{array}{l}\text { vs. WM } \\
\text { (1) RR } 1.22(1.08,1.37), p=0.001 \\
\text { (2) MD }-3.29(-8.22,1.64) \\
p=0.19 \\
\text { Cupping + AT vs. AT } \\
\text { (1) RR 1.05 }(0.99,1.12), p=0.13\end{array}$ & $\begin{array}{l}\ldots \text { improves } \ldots \\
\text { effect of migraine } \\
\ldots\end{array}$ & $\begin{array}{l}\text { Potentially } \\
\text { effective }\end{array}$ \\
\hline
\end{tabular}


Table 1. Cont

\begin{tabular}{|c|c|c|c|c|c|c|c|c|}
\hline $\begin{array}{l}\text { First Author } \\
\text { (year) (Ref) } \\
\text { Country }\end{array}$ & $\begin{array}{l}\text { Condition } \\
\text { Search Date } \\
\text { No. of Primary Studies }\end{array}$ & $\begin{array}{l}\text { Cupping } \\
\text { Therapy }\end{array}$ & Comparator & Outcome & $\begin{array}{l}\text { Overall Risk } \\
\text { of Bias }\end{array}$ & $\begin{array}{l}\text { Effect Estimates for Main } \\
\text { Outcomes (Meta-Analysis) }\end{array}$ & $\begin{array}{l}\text { Conclusion } \\
\text { (Quoted from the } \\
\text { Original Paper) }\end{array}$ & $\begin{array}{l}\text { Overall } \\
\text { Confidence }\end{array}$ \\
\hline $\begin{array}{l}\text { Xing } \\
(2020) \\
{[17]} \\
\text { China }\end{array}$ & $\begin{array}{l}\text { Plaque psoriasis } \\
\text { Mar } 2020 \\
16 \text { RCTs }\end{array}$ & $\begin{array}{l}\text { Moving } \\
\text { cupping }\end{array}$ & $\begin{array}{l}\text {-Oral Chinese } \\
\text { medicine } \\
\text {-Placebo } \\
\text {-WM }\end{array}$ & $\begin{array}{l}\text { (1) Recovery rate } \\
\text { (2) Recurrence rate } \\
\text { (3) VAS }\end{array}$ & High & $\begin{array}{l}\text { (1) SMD }-1.22(-1.58,-0.85) \\
p<0.00001 \\
\text { (2) RR } 0.33(0.16,0.68), p=0.003 \\
\text { (3) WMD }-0.27(-0.71,0.17) \\
p=0.22\end{array}$ & $\begin{array}{l}\ldots \text { could be an } \\
\text { effective ... }\end{array}$ & $\begin{array}{l}\text { Potentially } \\
\text { effective }\end{array}$ \\
\hline $\begin{array}{l}\text { Xiao } \\
(2020) \\
{[25]} \\
\text { China }\end{array}$ & $\begin{array}{l}\text { Chronic urticaria } \\
\text { May } 2019 \\
12 \text { RCTs }\end{array}$ & All types & $\begin{array}{l}-\mathrm{AT} \\
-\mathrm{WM}\end{array}$ & $\begin{array}{l}\text { (1) Effective rate } \\
\text { (2) Recurrence rate }\end{array}$ & High & $\begin{array}{l}\text { Wet cupping vs. WM } \\
\text { (1) RR } 1.10(0.97,1.25), p=0.14 \\
\text { (2) RR } 0.56(0.23,1.36), p=0.20 \\
\text { Cupping + WM vs. WM } \\
\text { (1) RR } 1.18(1.01,1.39), p=0.03 \\
\text { (2) RR } 0.52(0.32,0.84), p=0.007\end{array}$ & $\begin{array}{l}\ldots \text { it may } \\
\text { enhance the } \\
\text { efficacy }\end{array}$ & $\begin{array}{l}\text { Potentially } \\
\text { effective }\end{array}$ \\
\hline
\end{tabular}

AT + cupping vs. AT

(1) OR $2.28(1.56,3.32)$,

$p<0.0001$

(2) SMD $-0.21(-0.36,-0.06)$,

$p=0.007$

(3) SMD $-0.69(-0.85,-0.54)$

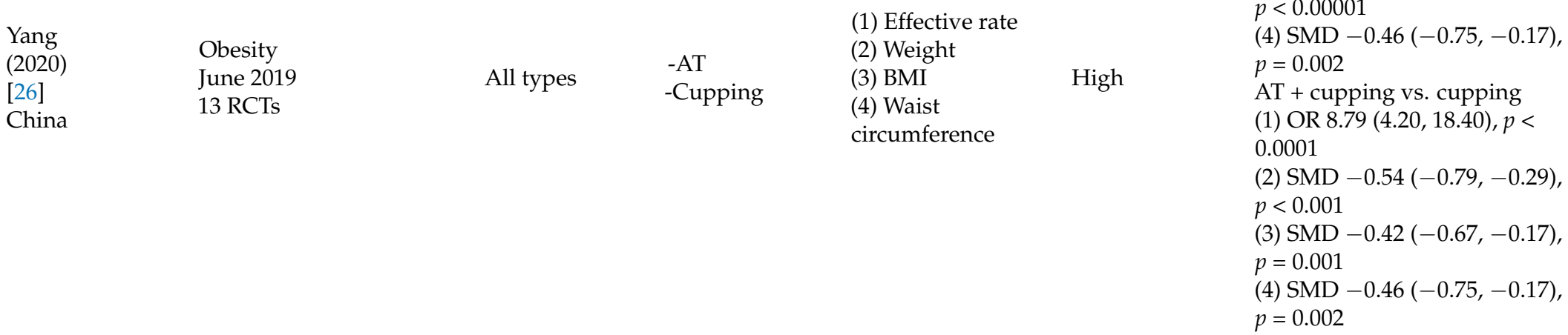

Insufficient ... Unclear

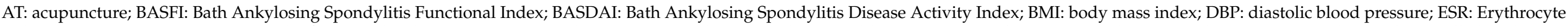

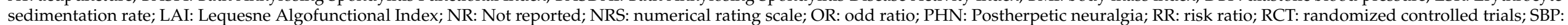

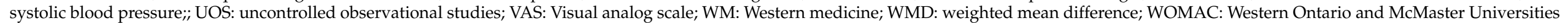
Osteoarthritis Index. 


\subsection{Quality of the Included Systematic Reviews}

Regarding the quality assessments of the overall confidence level for each SR, most reviews showed a moderate to critically low quality. All the reviews included comprehensive searches, interpreted the results based on the risk of bias assessment results, and reported any conflicts of interest. No reviews mentioned the study lists that were excluded or provide a satisfactory explanation for the heterogeneity in their results. The overall confidence was rated as "moderate" for six SRs [10,17-19,22,25], "low" for six SRs [9,11,20,21,24,26], and as "critically low" for one SR [23] (Table 2).

Table 2. Quality assessment of all included systematic reviews using AMSTAR 2.

\begin{tabular}{|c|c|c|c|c|c|c|c|c|c|c|c|c|c|c|c|c|c|}
\hline Study ID & 1 & 2 & 3 & 4 & 5 & 6 & 7 & 8 & 9 & 10 & 11 & 12 & 13 & 14 & 15 & 16 & $\begin{array}{l}\text { Rating Overall } \\
\text { Confidence * }\end{array}$ \\
\hline Moura (2018) [9] & Yes & No & Yes & Yes & No & No & No & Yes & Yes & Yes & Yes & Yes & Yes & No & No & No & Critically low \\
\hline Kim (2018) [10] & Yes & Yes & Yes & Yes & Yes & Yes & No & Yes & Yes & Yes & Yes & Yes & Yes & No & No & Yes & Moderate \\
\hline $\mathrm{Lu}(2018)$ [11] & Yes & No & Yes & Yes & Yes & Yes & No & Yes & Yes & No & Yes & Yes & Yes & No & No & Yes & Low \\
\hline $\mathrm{Ma} \mathrm{(2018)} \mathrm{[18]}$ & Yes & Yes & Yes & Yes & Yes & No & No & Yes & Yes & Yes & Yes & Yes & Yes & No & No & Yes & Low \\
\hline Li (2017) [19] & Yes & Yes & Yes & Yes & Yes & Yes & No & Yes & Yes & Yes & Yes & Yes & Yes & No & No & Yes & Moderate \\
\hline Zhang (2017) [20] & Yes & Yes & Yes & Yes & Yes & Yes & No & Yes & Yes & Yes & Yes & Yes & Yes & No & No & Yes & Low \\
\hline Cao (2010) [21] & No & No & Yes & Yes & No & Yes & No & Yes & Yes & Yes & Yes & No & Yes & No & Yes & Yes & Critically low \\
\hline Cao (2012) [22] & No & No & Yes & Yes & Yes & Yes & No & Yes & Yes & Yes & Yes & No & Yes & No & Yes & Yes & Critically low \\
\hline Lee (2010a) [23] & Yes & No & No & Yes & No & Yes & No & Yes & Yes & Yes & No-MA & No-MA & Yes & No-MA & No-MA & Yes & Critically low \\
\hline Seo (2018) [24] & Yes & Yes & Yes & Yes & Yes & No & No & Yes & Yes & No & Yes & No & Yes & Yes & No & No & Low \\
\hline Xing (2020) [17] & Yes & Yes & Yes & Yes & Yes & Yes & No & Yes & Yes & Yes & Yes & Yes & Yes & Yes & Yes & Yes & Moderate \\
\hline Xiao (2020) [25] & Yes & No & Yes & Yes & Yes & Yes & No & Yes & Yes & Yes & Yes & Yes & Yes & Yes & No & Yes & Moderate \\
\hline Yang (2020) [26] & Yes & No & Yes & No & Yes & Yes & No & Yes & Yes & Yes & Yes & Yes & Yes & No & Yes & No & Low \\
\hline
\end{tabular}

AMSTAR 2: A MeaSurement Tool to Assess systematic Reviews 2; No-MA: No meta-analysis conducted. 1. components of PICO/2. established prior to the conduct of the review/3. explain their selection of the study designs/4. comprehensive search/5. duplicate selection/6. duplicate extraction/7. list of excluded studies and justify the exclusions/8. describe the included studies in adequate detail/9. use a satisfactory technique for assessing the risk of bias (RoB)/10. report on the sources of funding/11. use appropriate methods for statistical combination of results/12. assess the potential impact of RoB in individual studies on the results/13. account for RoB in individual studies when interpreting/discussing the results of the review?/14. Did the review authors provide a satisfactory explanation for, and discussion of, any heterogeneity observed in the results of the review/15. Publication bias assessed/16. Include conflict of interest * AMSTAR2 was used to critically appraise the reporting quality of each included SR. The overall confidence of each SR was graded as "high", "moderate", "low" or "critically low".

\subsection{Effectiveness}

For the overlapping diseases, overall confidence was considered. The conclusions were reflected in the individual SRs and confirmed through an internal review.

\subsubsection{Evidence of a Positive Effect}

The effects of cupping, as indicated by statistically significant pooled treatment effects in SRs, were determined based on a substantial number of research studies that included findings on low back pain.

\subsubsection{Evidence of a Potentially Positive Effect}

Promising effects of cupping, as indicated by statistically significant pooled treatment effects in SRs, were determined based on a substantial number of research studies on conditions including ankylosing spondylitis, knee osteoarthritis, neck pain, herpes zoster, migraine, plaque psoriasis, and chronic urticaria.

\subsubsection{Evidence of Unclear}

The map includes a small number of SRs that provided evidence of the potential lack of effectiveness of cupping in treating clinical conditions, e.g., cervical spondylosis, lateral femoral cutaneous neuritis, scapulohumeral periarthritis, facial paralysis, acne, stroke rehabilitation, hypertension, and obesity, based on more than one included study.

\subsection{Evidence Map}

Figure 2 visualized the results of the evidence mapping. The evidence map shows the evidence of each included SR in the form of bubbles. As described in the prior Materials and Methods section, each bubble label represents the medical condition investigated in 
that review. The bubble size indicates the total sample size of a particular medical condition studied for the effectiveness of cupping as similar primary studies could be included in multiple SRs. Each bubble was plotted according to the strength of the findings for cupping (color), the effect of cupping ( $x$-axis), and the number of primary studies ( $y$-axis). The details of the included SRs were provided in Tables 1 and 2.

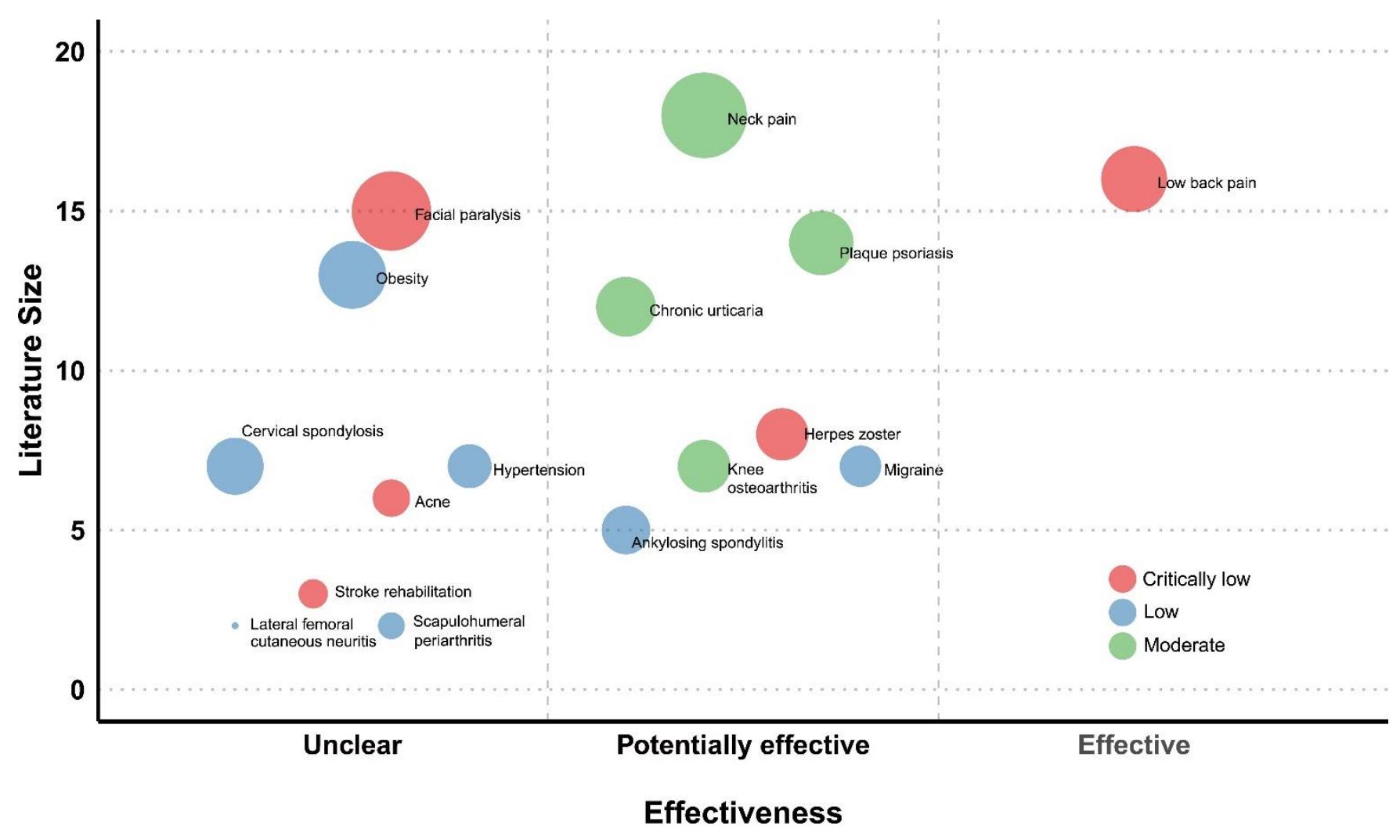

Figure 2. Evidence map of cupping therapy.

All included SRs concluded that cupping did not improve the outcomes of interest; however, the number of existing studies was small in all of the identified topic areas. The evidence mapping results showed that only a restricted number of cupping therapies were assessed with inconclusive clinical evidence, indicating the necessity of additional primary research in this area (Figure 2).

\section{Discussion}

The evidence map visualizations show the presence and absence of evidence for cupping therapy based on 13 published SRs and identified the evidence gaps relating to the details of the cupping therapy in particular. The studies included in our evidence map provide a very extensive and broad overview of the evidence on cupping therapy published between 2010 and 2020. Our evidence map also highlights the areas where metaanalyses have reported positive or unclear results, in the meantime showing the research concentration and volume. This evidence mapping process shows a range of evidence on cupping for any condition. Cupping has been clinically utilized and evaluated for a wide range of clinical conditions. Several identified SRs have addressed a very broad topic such as low back pain even though they included a large number of RCTs. Additionally, evidence on the role of cupping for specific clinical conditions is also very limited due to the small number of published studies.

Even though evidence maps can provide only a broad overview of research areas, our review concluded that more rigorous research on the clinical effectiveness of cupping 
is needed across various clinical topic areas. The duration and frequency of the cupping interventions in the cited studies have yet to be evaluated and need to be studied further. Furthermore, such inconclusive evidence also warrants more research.

The findings of seven reviews showed evidence of potential benefits of cupping for conditions including neck pain, knee osteoarthritis, plaque psoriasis, chronic urticarial, ankylosing spondylitis, herpes zoster, and migraine. Many studies have provided evidence concerning the effectiveness of cupping in treating certain clinical indications. Cupping therapy is a traditional method and is currently used in the treatment of a broad range of medical and health conditions. Nevertheless, the mechanism of action of cupping remains unclear and is yet to be fully understood [27].

Evidence maps are one of many tools and information sources to support evidence. It is similar to systematic review methods that are reproducible and transparent. However, while SRs target specific research questions, evidence mapping focuses on the nature, volume, and characteristics of the research to identify, describe and summarize what is known [28]. The creation and publication of evidence maps consist of graphically representing the highest quality evidence found, analyzing and categorizing it, and linking it with the bibliographic records and full texts of the studies to facilitate access to information for everyone interested.

This evidence map has described the research focus, which was reported in the existing SRs, and identified the gaps in evidence to pinpoint areas that should be prioritized in future research. However, this evidence map is unable to answer more refined questions, such as the most adequate cupping therapy for specific applications and the differences between health services. To further advance our evidence-based knowledge of cupping, we should collect more data on the effectiveness of cupping across and within each clinical condition and patient population through meta-analyses across primary studies. Additionally, the large number of topic areas that were classified as having unclear evidence warrants additional primary studies. More studies have been published in some of the topic areas included in the unclear evidence category, and the currently available SRs need to be updated. As there might be other efficient ways of drawing evidence maps, further research should also include developing evidence maps of other research designs.

In conclusion, cupping has been applied in a variety of clinical areas, and for a few of these, SRs have demonstrated statistically significant results. This evidence map provides a very broad overview of the evidence base, indicating the areas in which research has been conducted. The evidence map provides a visual overview of the cupping research volume and content. Evidence mapping can facilitate the transfer of knowledge from researchers to policymakers and promote research on musculoskeletal pain (as low back pain, neck pain, and knee osteoarthritis) and skin disease (plaque psoriasis).

Supplementary Materials: The following are available online at https://www.mdpi.com/article/ 10.3390/jcm10081750/s1, Supplement 1: Search strategy. Supplement 2. CNKI Chinese database. Supplement 3. Korea Med Korean database.

Author Contributions: Conceptualization, T.Y.C. and M.S.L.; methodology, T.Y.C., J.H.J. and M.S.L.; software, T.Y.C. and B.K.; validation, T.Y.C. and M.S.L.; formal analysis, T.Y.C. and M.S.L.; investigation, T.Y.C., L.A. and M.S.L.; resources, L.A. and M.S.L.; data curation, T.Y.C. and B.K.; writingoriginal draft preparation, T.Y.C. and M.S.L.; writing-review and editing, L.A., J.H.J. and B.K.; visualization, T.Y.C. and B.K.; supervision, M.S.L.; project administration, M.S.L.; funding acquisition, M.S.L. All authors have read and agreed to the published version of the manuscript.

Funding: This research was funded by Korea Institute of Oriental Medicine (KSN2013210 and KSN1522120).

Institutional Review Board Statement: Not applicable.

Informed Consent Statement: Not applicable.

Data Availability Statement: Data sharing not applicable. 
Conflicts of Interest: The authors declare no conflict of interest. The funders had no role in the design of the study; in the collection, analyses, or interpretation of data; in the writing of the manuscript, or in the decision to publish the results.

\section{References}

1. Lin, K.C.; Chen, M.L.; Yeh, M.L.; Chen, Y.L.; Chou, P. Prevalence, pattern, and predictors of use of complementary and alternative medicine in Taiwan. Taiwan J. Public Health 2009, 28, 53-68.

2. Farhadi, K.; Schwebel, D.C.; Saeb, M.; Choubsaz, M.; Mohammadi, R.; Ahmadi, A. The effectiveness of wet-cupping for nonspecific low back pain in Iran: A randomized controlled trial. Complement. Ther. Med. 2009, 17, 9-15. [CrossRef]

3. Aboushanab, T.S.; AlSanad, S. Cupping Therapy: An Overview from a Modern Medicine Perspective. J. Acupunct. Meridian Stud. 2018, 11, 83-87. [CrossRef] [PubMed]

4. Mohsen, M.K.; Reza, M.; Mohammad, A.; Keyvan, H.M. Wet-Cupping Is Effective on Persistent Nonspecific Low Back Pain: A Randomized Clinical Trial. Chin. J. Integr. Med. 2018. [CrossRef]

5. Saha, F.J.; Schumann, S.; Cramer, H.; Hohmann, C.; Choi, K.E.; Rolke, R.; Langhorst, J.; Rampp, T.; Dobos, G.; Lauche, R. The Effects of Cupping Massage in Patients with Chronic Neck Pain-A Randomised Controlled Trial. Complement. Med. Res. 2017, 24, 26-32. [CrossRef]

6. Teut, M.; Kaiser, S.; Ortiz, M.; Roll, S.; Binting, S.; Willich, S.N.; Brinkhaus, B. Pulsatile dry cupping in patients with osteoarthritis of the knee-A randomized controlled exploratory trial. BMC Complement. Altern. Med. 2012, 12, 184. [CrossRef] [PubMed]

7. Chirali, I.Z. Cupping Therapy, 3rd ed.; Elsevier: Philadelphia, PA, USA, 2014.

8. Lee, M.S.; Kim, J.I.; Ernst, E. Is cupping an effective treatment? An overview of systematic reviews. J. Acupunct. Meridian Stud. 2011, 4, 1-4. [CrossRef]

9. Moura, C.C.; Chaves, E.C.L.; Cardoso, A.; Nogueira, D.A.; Correa, H.P.; Chianca, T.C.M. Cupping therapy and chronic back pain: Systematic review and meta-analysis. Revista Latino-Americana Enfermagem 2018, 26, e3094. [CrossRef]

10. Kim, S.; Lee, S.H.; Kim, M.R.; Kim, E.J.; Hwang, D.S.; Lee, J.; Shin, J.S.; Ha, I.H.; Lee, Y.J. Is cupping therapy effective in patients with neck pain? A systematic review and meta-analysis. BMJ Open 2018, 8, e021070. [CrossRef] [PubMed]

11. Lu, S.; Du, S.; Fish, A.; Tang, C.; Lou, Q.; Zhang, X. Wet cupping for hypertension: A systematic review and meta-analysis. Clin. Exp. Hypertens. 2018, 474-480. [CrossRef] [PubMed]

12. Cao, H.J.; Han, M.; Zhu, X.S.; Liu, J.P. An overview of systematic reviews of clinical evidence for cupping therapy. J. Tradit. Chin. Med. Sci. 2015, 2, 3-10. [CrossRef]

13. Wang, D.D.; Shams-White, M.; Bright, O.J.; Parrott, J.S.; Chung, M. Creating a literature database of low-calorie sweeteners and health studies: Evidence mapping. BMC Med. Res. Methodol. 2016, 16, 1. [CrossRef] [PubMed]

14. Miake-Lye, I.M.; Hempel, S.; Shanman, R.; Shekelle, P.G. What is an evidence map? A systematic review of published evidence maps and their definitions, methods, and products. Syst. Rev. 2016, 5, 28. [CrossRef] [PubMed]

15. Hempel, S.; Taylor, S.L.; Solloway, M.R.; Miake-Lye, I.M.; Beroes, J.M.; Shanman, R.; Booth, M.J.; Siroka, A.M.; Shekelle, P.G. VA evidence-based synthesis program reports. In Evidence Map of Acupuncture; Department of Veterans Affairs: Washington, DC, USA, 2014.

16. Shea, B.J.; Reeves, B.C.; Wells, G.; Thuku, M.; Hamel, C.; Moran, J.; Moher, D.; Tugwell, P.; Welch, V.; Kristjansson, E.; et al. AMSTAR 2: A critical appraisal tool for systematic reviews that include randomised or non-randomised studies of healthcare interventions, or both. BMJ 2017, 358, j4008. [CrossRef] [PubMed]

17. Xing, M.; Ding, X.; Zhang, J.; Kuai, L.; Ru, Y.; Sun, X.; Ma, T.; Miao, X.; Liu, L.; Wang, Y.; et al. Moving cupping therapy for plaque psoriasis: A PRISMA-compliant study of 16 randomized controlled trials. Medicine 2020, 99, e22539. [CrossRef] [PubMed]

18. Ma, S.Y.; Wang, Y.; Xu, J.Q.; Zheng, L. Cupping therapy for treating ankylosing spondylitis: The evidence from systematic review and meta-analysis. Complement. Ther. Clin. Pract. 2018, 32, 187-194. [CrossRef]

19. Li, J.Q.; Guo, W.; Sun, Z.G.; Huang, Q.S.; Lee, E.Y.; Wang, Y.; Yao, X.D. Cupping therapy for treating knee osteoarthritis: The evidence from systematic review and meta-analysis. Complement. Ther. Clin. Pract. 2017, 28, 152-160. [CrossRef]

20. Zhang, Y.J.; Cao, H.J.; Li, X.L.; Yang, X.Y.; Lai, B.Y.; Yang, G.Y.; Liu, J.P. Cupping therapy versus acupuncture for pain-related conditions: A systematic review of randomized controlled trials and trial sequential analysis. Chin. Med. 2017, 12, 21. [CrossRef] [PubMed]

21. Cao, H.; Zhu, C.; Liu, J. Wet cupping therapy for treatment of herpes zoster: A systematic review of randomized controlled trials. Altern. Ther. Health Med. 2010, 16, 48-54.

22. Cao, H.; Li, X.; Liu, J. An updated review of the efficacy of cupping therapy. PLoS ONE 2012, 7, e31793. [CrossRef]

23. Lee, M.S.; Choi, T.Y.; Shin, B.C.; Han, C.H.; Ernst, E. Cupping for stroke rehabilitation: A systematic review. J. Neurol. Sci. 2010, 294, 70-73. [CrossRef] [PubMed]

24. Seo, J.H. Cupping Treatment for Migraine: A Systematic Review of Randomized Controlled Trial; Graduate School of Wonkwang University: Iksan, Korea, 2018.

25. Xiao, X.J.; Zhang, L.X.; Shi, Y.Z.; Yao, J.P.; Cao, W.; Liu, Y.; Zou, Z.H.; Zhou, S.Y.; Chen, M.L.; Li, C.X.; et al. Cupping therapy for patients with chronic urticaria: A systematic review and meta-analysis. J. Integr. Med. 2020, 18, 303-312. [CrossRef]

26. Yang, J.; Ren, X.; Yang, Q.; Zhang, W.; Gao, R. Efficacy and safety of acupuncture combined with cupping in the treatment of simple obesity: A meta-analysis. Mod. Tradit. Chin. Med. Mater. Mater. World Sci. Technol. 2020, 22, 3358-3366. 
27. Al-Bedah, A.M.N.; Elsubai, I.S.; Qureshi, N.A.; Aboushanab, T.S.; Ali, G.I.M.; El-Olemy, A.T.; Khalil, A.A.H.; Khalil, M.K.M.; Alqaed, M.S. The medical perspective of cupping therapy: Effects and mechanisms of action. J. Tradit. Complement. Med. 2019, 9, 90-97. [CrossRef] [PubMed]

28. Wolffe, T.A.M.; Whaley, P.; Halsall, C.; Rooney, A.A.; Walker, V.R. Systematic evidence maps as a novel tool to support evidencebased decision-making in chemicals policy and risk management. Environ. Int. 2019, 130, 104871. [CrossRef] 